\title{
Partially Hydrolyzed Guar Gum, an Organoleptically-clean Prebiotic Fiber Ingredient for Dietary Supplements
}

\author{
Ji $\mathrm{Li}^{1 *}$ and Bing $\mathrm{Hu}^{2}$ \\ ${ }^{1}$ Nutraceutical Corporation, USA \\ ${ }^{2}$ College of Food Science and Technology, Nanjing Agricultural University, China \\ *Corresponding author: Ji Li, Nutraceutical Corporation, 550 West 2nd Street, Ogden, UT 84404, USA
}

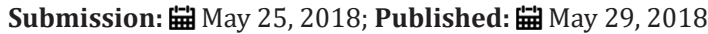

\begin{abstract}
Partially hydrolyzed guar gum (PHGG) is a natural prebiotic fiber, which can be produced through controllable enzymatic hydrolysis of guar gum. Compared with guar gum, PHGG has a smaller molecular weight and lower viscosity. Besides, PHGG enjoys quite a few supplement ingredients' advantages including clean taste and texture, storage stability, and resistance to heat, acid, salt, and digestive enzyme. Guar gum-based prebiotic fiber products on the current market are monotonous, simple, and embedded with sparse brands. We put forward and invoke that product development team should make full use of different actives, flavors, dosages, and processes to diversify the guar gum product profile on the market of dietary supplement.
\end{abstract}

Keywords: Partially hydrolyzed guar gum; Prebiotic; Fiber; Diversification

\section{Opinion}

Partially hydrolyzed guar gum (PHGG) is a natural ingredient produced through controllable enzymatic hydrolysis of guar gum [1]. Various enzymes such as mannanse, pectinase, cellulose etc. can be used to chop the mannose-mannose linkage and generate PHGG [2]. Guar gum individually, is a galactomannan polysaccharide composed of $(1 \rightarrow 4)-\beta-D$ mannopyranosyl units and $(1 \rightarrow 6)-\alpha-D$ galactopyranosyl units [3]. The plant of guar bean is exclusively grown in India, Pakistan, U.S., Australia, and Africa. Among those countries, United States has manufactured approximately 10,000 tons of guar in the past five years. Major demand of guar gum comes from industrial applications, including: stabilizer or thickener in consumer goods such as juice, ice cream, sauce, salad dressing, toothpaste, and shampoo; binder and disintegrant in supplement such as swallow able tablet [4,5]; and waterproofing agent used with ammonium nitrate in explosive products. In addition to those uses, the high viscosity of guar gum due to its high molecular weight makes it challenging to be integrated into more popular products (e.g., beverage, drink mix), and then depreciates its own value as supplement.

Partially hydrolyzed guar gum (PHGG) has a smaller molecular weight and less viscosity than original guar gum. PHGG, the product of guar hydrolysis, is shelf-stable, soluble at commonly-intake $\mathrm{pH}$, resistant to heat, acid, salt, digestive enzyme, and with a bland, non- gummy texture, the properties of which pave the way for making more guar-involved products for healthcare. It has been reported that PHGG plays a role in maintaining and improving the gastrointestinal health as a better resource of dietary fiber. For instance, Tuohy et al. [5] reported a clinical study of prebiotic potential of PHGG and fructooligosaccharides (FOS) in biscuits [6]. The study results showed that bifidobacteria, the gut microflora, largely increased in number upon the intake of the PHGG/FOS-included biscuits compared with pre-treatment and placebo population levels. It then took 7 days for the population of bifidobacterial to decrease back to pretreatment level since the last intake of the PHGG/FOS-

included biscuits, which suggested a long period of probiotics supplementation. In addition to increasing probiotics, PHGG can effective alleviate symptoms in constipation-predominant and diarrhea-predominant forms of Irritable bowel syndrome, and reduce abdominal pains [7]. Besides, Minekus et al. [7] found that the PHGG could decrease the bio-accessibility of fat and cholesterol with a dose-dependent manner through the depletion flocculation mechanism [8]. This piece of report unveils a synergistic effect of PHGG to potentially clean the gut and reduce the risk of cardiovascular disease.

Besides, with an organoleptically-clean profile, PHGG is suitable to be a filler-binder in various supplement products such 
as 2-piece hard-shell gelatin or vegetable capsules, powders, and chewable tablets. For instance, Table 1 displays one powder example of PHGG-inulin fiber drink mix - strawberry flavor, which is not a product on the market yet. In this formula, PHGG together with inulin provide us with a $6 \mathrm{Gm}$ dietary fiber per dose, and the pleasant fruity strawberry note greatly enhances the sensational experience of this drink mix product.

Table 1: Partially hydrolyzed guar gum (PHGG)-inulin fiber drink mix powder with strawberry flavour.

\begin{tabular}{|c|c|c|}
\hline Ingredient & $\begin{array}{c}\text { Ingredient Amount, } \\
\mathbf{G m}\end{array}$ & $\mathbf{\%}$ \\
\hline PHGG & 2.5 & $31.31 \%$ \\
\hline Inulin & 4.21 & $52.73 \%$ \\
\hline Stevia & 0.07 & $0.81 \%$ \\
\hline Luo Han Extract & 0.24 & $3.01 \%$ \\
\hline Strawberry Flavor & 0.49 & $6.14 \%$ \\
\hline Citric Acid - Fine & 0.06 & $0.75 \%$ \\
\hline Beet Root Powder & 0.02 & $0.25 \%$ \\
\hline Xylitol Fine & 0.4 & $5.01 \%$ \\
\hline Total & 7.99 & $100.00 \%$ \\
\hline
\end{tabular}

Meanwhile, different health manufacturers such as GNC, NOW Foods, and Swanson are producing guar gum or partially hydrolyzed guar gum (PHGG) fiber products as part of their supplements' line. Most of those guar fiber products on the market are in powder dosage with dietary fiber claims range from $3 \mathrm{Gm}$ per dose to $15 \mathrm{Gm}$ per dose. Swanson Vitamin offers a guar gum capsule product in their category of digestive health and fiber, one of the very few capsule products on the market. However, most of those powder and capsule products with guar fiber are monotonous, some with merely single-ingredient. In other words, the diversification of guarderived fiber products are lacking on the current dietary supplement market. Expanding the product diversity is helpful to meet the dietary fiber demand of consumers from small to elder. Due to the fact that PHGG is an organoleptically-clean fiber and filler-binder as mentioned, it is convenient for product development to integrate this well-balanced ingredient into flavored powder drink mix along with other functional ingredients (e.g., fibers (the example in Table 1), branch-chain amino acids (BCAA) [9], and whey proteins [10]), chewable tablets, and etc. Various flavor options such as citrus fruity flavors, creamy vanilla flavors, and etc. can be utilized to boost the sensation of the entire supplement product, while the combination of PHGG with other supplements such as BCAA, whey protein, multi-vitamins and etc. enables the massive consumers to have an one-stop service of multi-functional fiber supplementation. Overally-speaking, it is suggested that product development team make efforts to produce more diversified supplements with partially hydrolyzed guar gum (PHGG). Different dosages, flavors, processes, and their combinations enable us to see more guar fiber or derivative products on the shelf. In future, the participation of novel excipients and even advanced technologies (e.g. 3D printing) might target accurate ingredient distribution and delivery.

\section{References}

1. Slavin JL, Greenberg NA (2003) Partially hydrolyzed guar gum: Clinical nutrition uses. Nutrition 19(6): 549-552.

2. Yoon SJ, Chu DC, Juneja LR (2008) Chemical and physical properties, safety and application of partially hydrolized guar gum as dietary fiber. J Clin Biochem Nutr 42(1): 1-7.

3. Mudgil D, Barak S, Khatkar BS (2014) Optimization of enzymatic hydrolysis of guar gum using response surface methodology. J Food Sci Technol 51(8): 1600-1605.

4. Krishnaiah YSR, Satyanarayana S, Rama Prasad YV, Raob NS (1998) Gamma scintigraphic studies on guar gum matrix tablets for colonic drug delivery in healthy human volunteers. J Control Release 55(2-3): 245-252.

5. Tuohy KM, Kolida S, Lustenberger AM, Gibson GR (2001) The prebiotic effects of biscuits containing partially hydrolysed guar gum and fructo-oligosaccharides - a human volunteer study. Brit J Nutr 86(3): 341348.

6. Giannini EG, Mansi C, Dulbecco P, Savarino V (2006) Role of partially hydrolyzed guar gum in the treatment of irritable bowel syndrome. Nutrition 22(3): 334-342.

7. Minekus M, Jelier M, Xiao JZ, Kondo S, Iwatsuki K, et al. (2005) Effect of partially hydrolyzed guar gum (PHGG) on the bioaccessibility of fat and cholesterol. Biosci Biotechnol Biochem 69(5): 932-938.

8. Lynch CJ, Adams SH (2014) Branched-chain amino acids in metabolic signalling and insulin resistance. Nat Rev Endocrinol 10(12): 723-736.

9. Ha E, Zemel MB (2003) Functional properties of whey, whey components, and essential amino acids: mechanisms underlying health benefits for active people (Review). J Nutr Biochem 14(5): 251-258.

10. Khaled SA, Burley JC, Alexander MR, Roberts CJ (2014) Desktop 3D printing of controlled release pharmaceutical bilayer tablets. Int J Pharm 461(1-2): 105-111.
Creative Commons Attribution 4.0 International License

For possible submissions Click Here

\section{Submit Article}

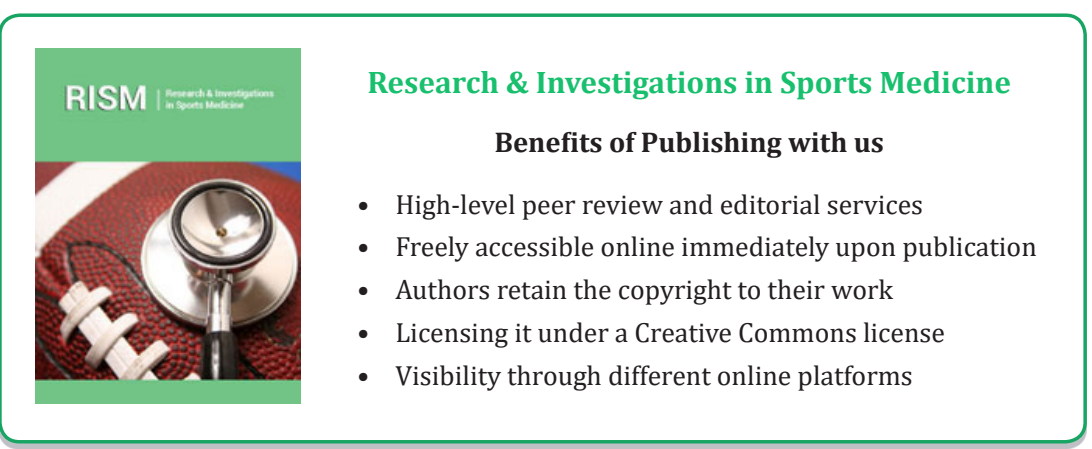

